\title{
Studies on the Role of Noradrenaline in the Pathogenesis of Hypertension
}

By

\author{
Kaoru Yoshinaga, Tatsuo Sato, Nozomu Ishida \\ From the Department of Internal Medicine, Tohokw University School \\ of Medicine, Sendai; Director : Prof. Dr. T. Torilia
}

(Received for publication, May 10, 1960)

\section{INTRODUCTION}

Noradrenaline is one of the powerful pressor substances which are found physiologically in the human body. Noradrenaline produces a type of hypertension that closely resembles essential hypertension, when it is infused in man. During the infusion, elevation in both the systolic and the diastolic pressure occurs and this pressor effect is proportionate to the quantity of administered noradrenaline. But no significant subjective symptoms are observed in the range of dosage between 0.01 and $0.3 \mu \mathrm{g} / \mathrm{kg} / \mathrm{min}$.

On the other hand, in the cases of pheochromocytoma, hypertension is caused by a large quantity of noradrenaline released from the chromafin cell tumor. Unfortunately, in about two-thirds of such cases the tumor induces sustained hypertension, so that the differential diagnosis between essential hypertension and pheochromocytoma is made very difficult.

Therefore, it is a very interesting problem to evaluate the role of noradrenaline in the pathogenesis of essential bypertension. The present study was undertaken for these reasons.

\section{EXPERIMENTS AND RESULTS}

\section{1) Blood-pressure response to the infusion of noradrenaline.}

According to Goldenberg's ${ }^{11}$ technique, the noradrenaline sensitivity tests were performed in 8 normotensives, 10 patients with ancomplicated essential hypertension and one patient with surgically verified pheochromocytoma.

The results are shown in Fig. 1, in which the dosage is plotted as abscissa and systolic blood pressure as ordinate, so the steeper the curve, the higher the sensitivity. It is obvious that the hypertensives are more sensitive to noradrenaline than normotensive subjects. These experimental data are similar to Goldenberg's' results. On the other hand, in the case of pheochromocytoma

吉永警，恢藤展男，不田望 


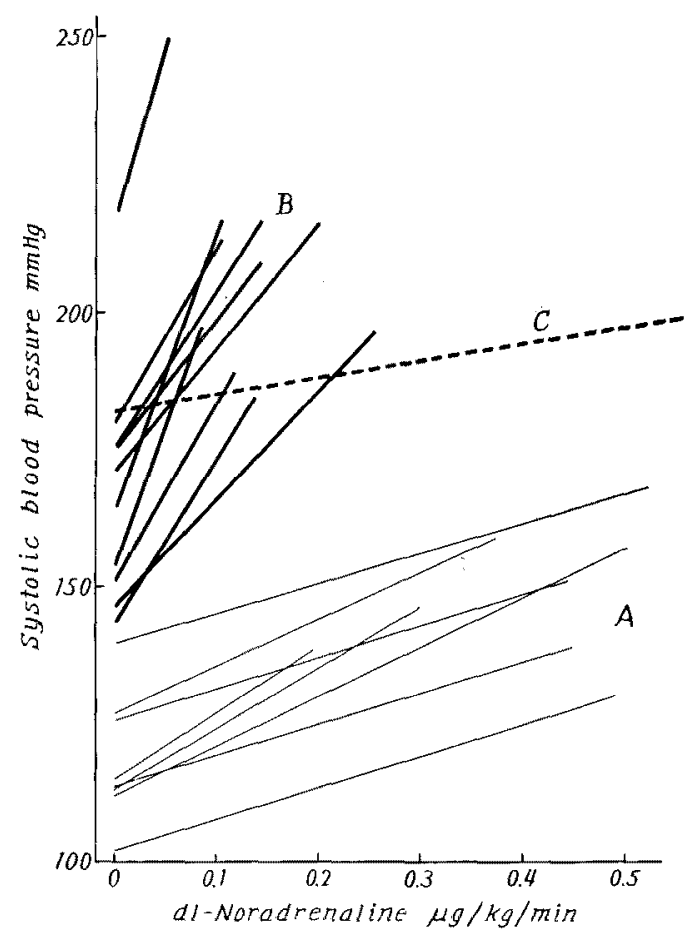

Fig. 1. The systolic-pressure responses to increasing doses of noradrenaline. A. normotensive group; B. hypertensive group ; C. pheochromocytoma.

this test showed lower sensitivity than in nomotensives. This seems to indicate that the high level of circulating pressor amine may actually obtund the responsiveness of the peripheral vascular system. If essential hypertension is caused by an excess of noradrenaline secretion, sensitivity to noradrenaline should be weakened as in this case of pheochromocytoma, but reverse results were obtained. Thus, our data suggest the genesis of essential hypertension is not due to an cxcess of noradrenaline released from sympathetic nerve endings or the adrenal medulla.

\section{2) Blood-pressure response to Regitine.}

Regitine ( $5 \mathrm{mg}$.) was administered intravenously to 68 cases with hypertension, and the relation between the change in blood pressure by this agent and the blood pressure before its injection was analysed. In this series, it was found that 2 cases of them had pheochromocytoma. Figure 2 demonstrates the typical response to Regitine in these two cases. Of the remaining 66 cases, 6 had renal hypertension, 3 Conn's syndrome and 57 essential hypertension.

Generally speaking, there was no relation between the response to Regitine 


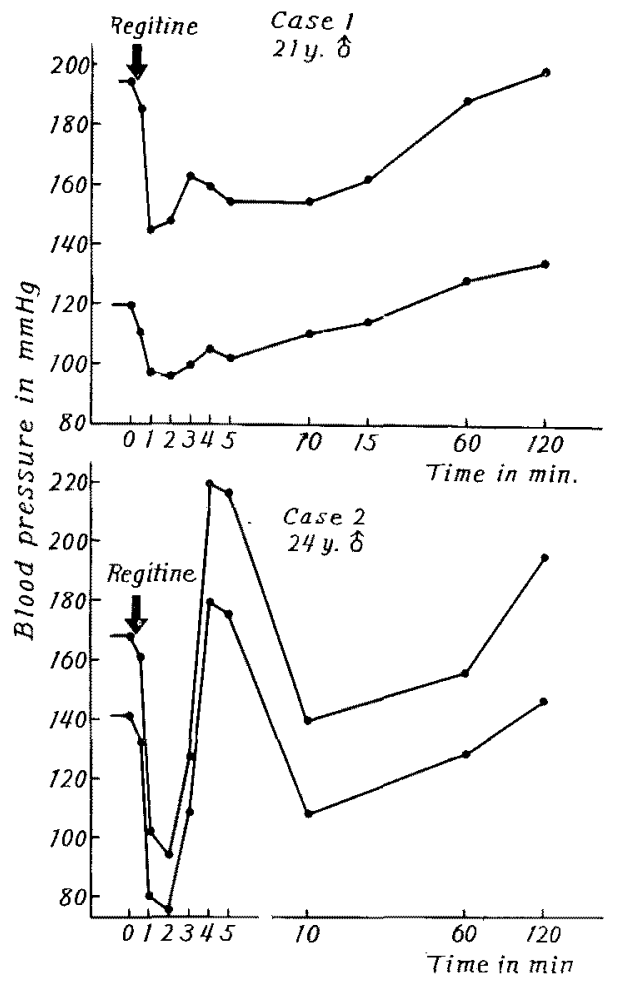

Fig. 2. Changes in blood pressure resulting from intravenous administration of $5 \mathrm{mg}$. of Regitine,

and blood pressure as shown in Figure 3.

Pharmacologically, Regitine possesses a marked adrenolytic effect on circulating pressor amine, while its sympathicolytic action is only very weak ${ }^{2}$. But the pressor effect of noradrenaline released from sympathetic nerve endings may be at least partially blocked by Regitine. Therefore, if hypertension is caused by an excess of noradrenaline, it may be expected that there is a parallel relation between the response to Regitine and the blood pressure.

Although it is questionable whether the response to Regitine may be taken as an indicator of noradrenaline production, the above results might indicate the cause of essential hypertension is not due to hypersecretion of noradrenaline.

\section{3) Quantity of noradrenaline in plasma and urine.}

a) Noradrenaline in plasma.

In order to evaluate the relation between hypertension and noradrenaline, venous blood plasma of 20 normotensives and 20 hypertensives were analysed for its content of noradrenaline according to Weil-Malherbe and Bone's ${ }^{31}$ method. 

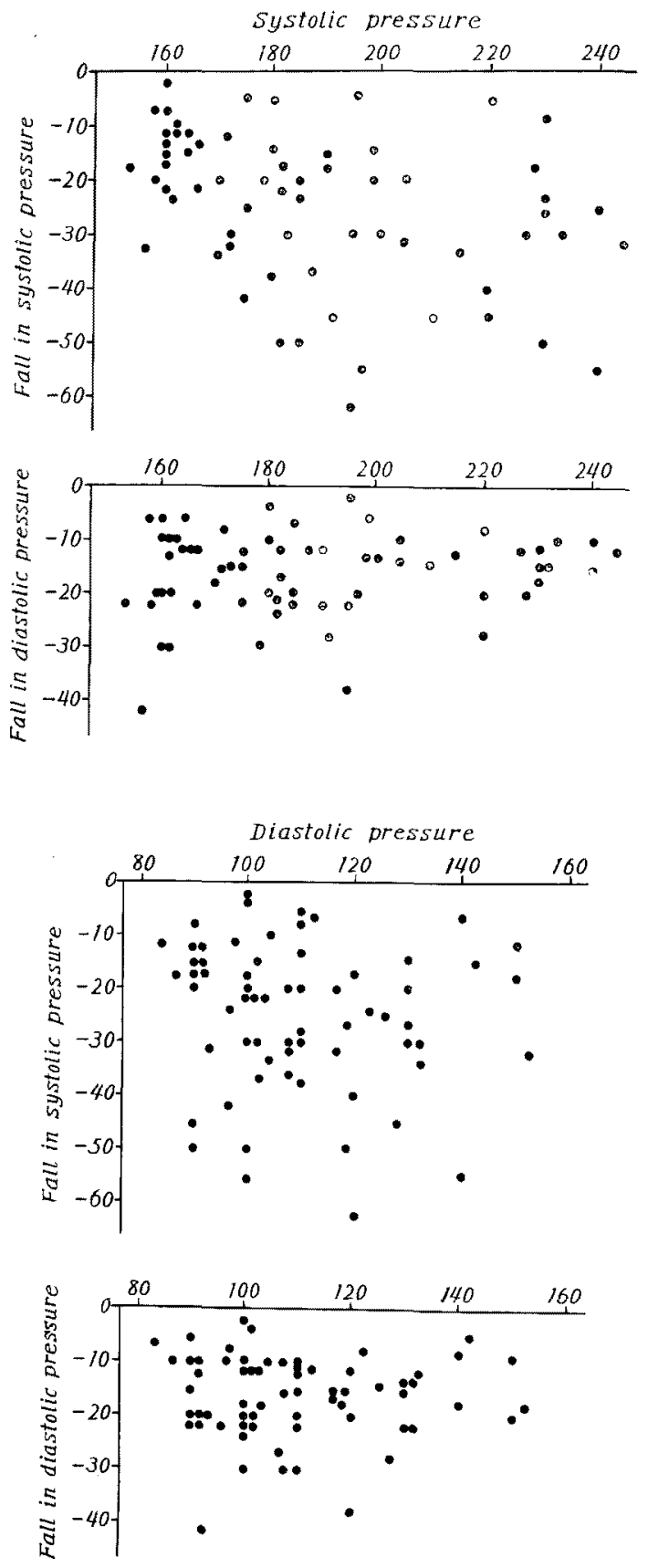

Fig. 3. The correlation between blood pressure and its response to Regitine. 

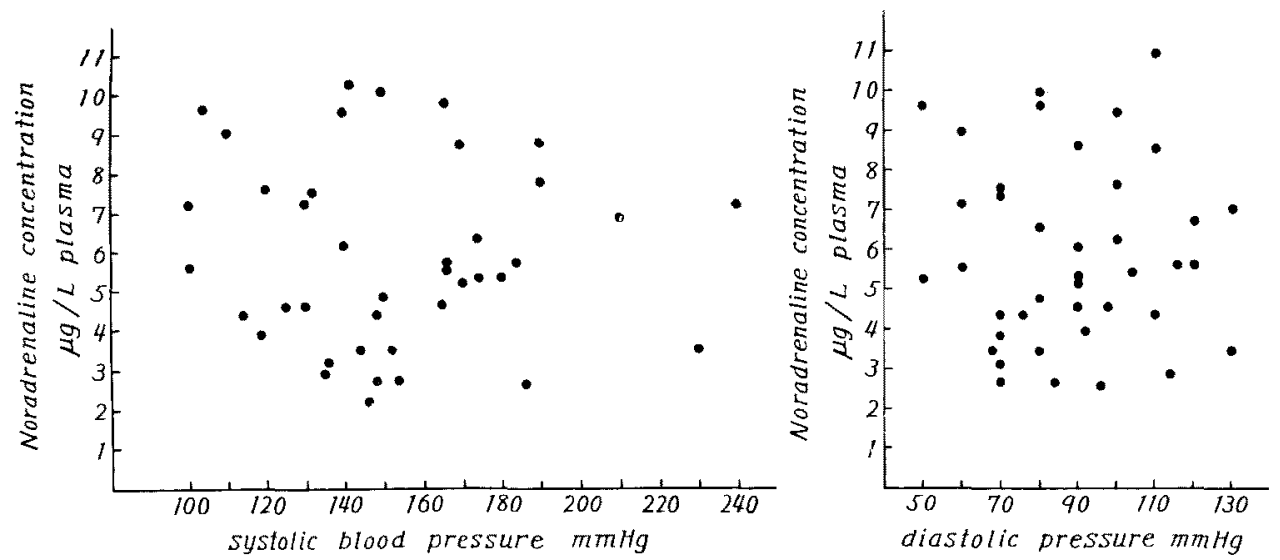

Fig. 4. Correlation diagram between blood pressure and noradrenaline concentration in plasma.

Blood samples were collected from the antecubital vein of patients lying quietly in bed at $10 \mathrm{a} . \mathrm{m}$. Immediately before sampling, the blood pressure was measured. As shown in Figure 4, there was no parallelism between noradrenaline concentration and blood pressure.

For the study of the role of noradrenaline in hypertension, it is the most reliable and necessary way to estimate accurately the concentrations of noradrenaline in plasma. However, as $\mathrm{we}^{4)}$ have reported previously, the method used here is not specific enough for catecholamines. The trihydroxyindole method has a very high specificity, but it can not be used in determining the contents of catecholamines in small amount of plasma, because the concentrations of these amines in venous plasma are extremely low.

\section{b) Noradrenaline in urine.}

Urinary noradrenaline was determined using the trihydroxyindole method described by Euler and Floding ${ }^{5}$. The subjects consisted of 20 normotensives and 20 hypertensives. They were asked to void at 2 p.m., then to lie quietly in bed for an hour, and to void again at 3 p.m. Their blood pressure was registered at 2.30 p.m. Thus the urine excreted during one hour was collected excluding the postural influence on noradrenaline excretion.

The results are shown in Figure 5. There was a tendency for the urinary noradrenaline to decrease with the rise of the blood pressure levels.

The technique used here is highly specific and its results were reported to be in good agreement with that of bioassay.

The amount of noradrenaline recovered in urine was reported ${ }^{6 /}$ to amount to only 2-3 per cent of the infused quantity, but this rate is similar in various conditions except in advanced renal damage. Therefore, the urinary noradrenaline can be considered as a good indicator of its endogenous production. The 

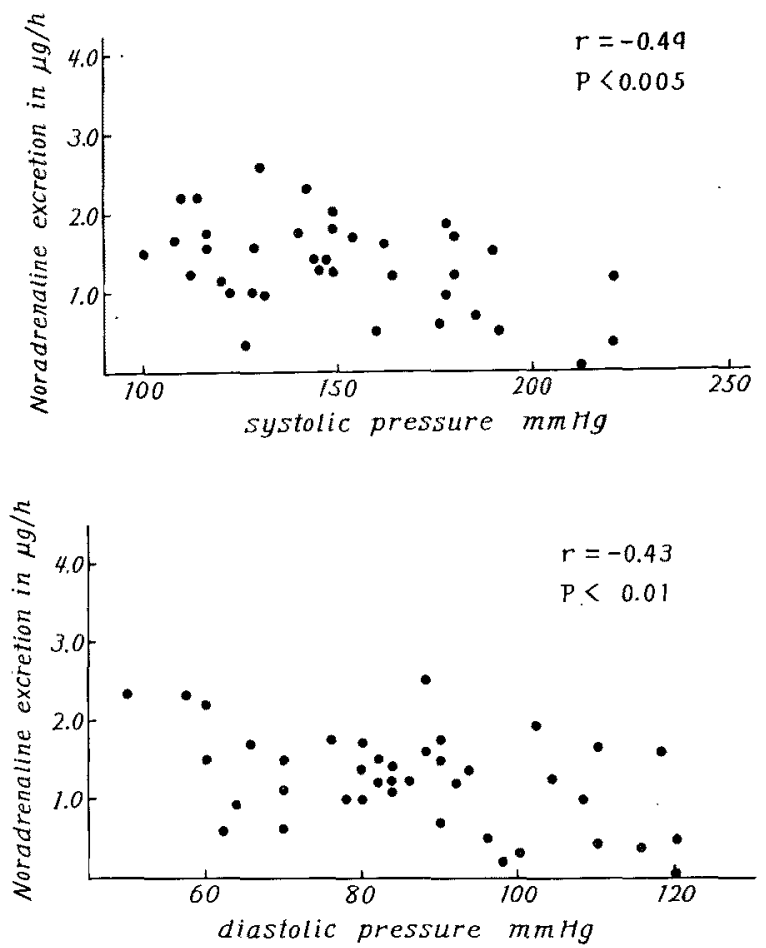

Fig. 5. The correlation between blood pressure and urinary noradrenaline at rest.

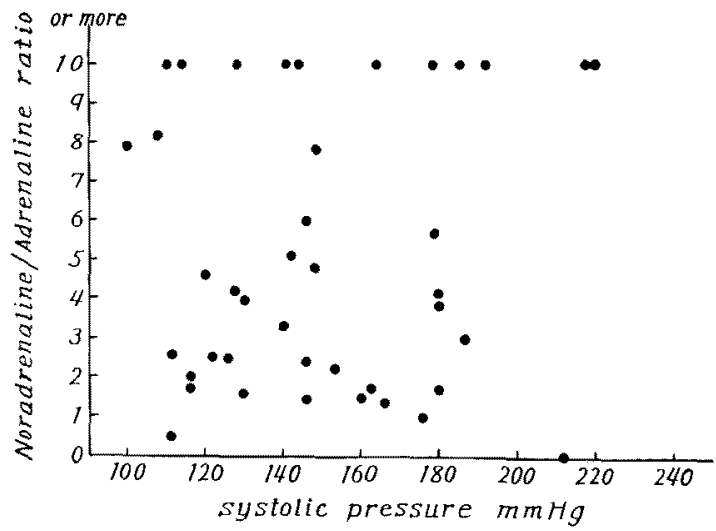

Fig. 6. The correlation between systolic blood pressure and noradrenaline/adrenaline ratio. 
above results also indicate that the cause of essential hypertension is not the excess of this pressor amine.

The ratio noradrenaline/adrenaline was calculated from the above mentioned data, and it was plotted against the systolic blood pressure (Figure 6). No correlation was noted between them.

However, since the standard deviation of repeated estimations of these amines was about $\pm 10 \%$ in our laboratory, and since a few per cent of noradrenaline was occasionally estimated as adrenaline and vice versa, the noradrenaline/ adrenaline ratio is not so exact that any decisive conclusions could be derivad from this calculated ratio. But the results described above do not support the Goldenberg's hypothesis ${ }^{1}$, that the increase of noradrenaline/adrenaline ratio is an important genetic factor in essential hypertension.

\section{DISCUSSION}

Many authors have investigated the role of noradrenaline in the pathogenesis of essential hypertention. The urinary excretion of noradrenaline in hypertensives was studied by Euler ${ }^{2)}$, Burn ${ }^{81}$, Goldenberg ${ }^{91}$ and so on, but they all found no difference between hypertensives and normotensives in this respect.

Sundin ${ }^{10)}$ found that the hypertensive patients excrete about the same amount of noradrenaline as the normotensives, but when the former are tilted to $75^{\circ}$ no increase takes place in noradrenaline excretion, while marked increase is regularly observed in the latter in the same condition. Recently $\mathrm{M} \phi l_{l} \mathrm{rr}^{111}$ reported that the amounts of urinary noradrenaline decrease with the rise of blood pressure levels.

Both Sundin and Moller concluded that the activity of the sympathetic nervous system of hypertensives is suppressed reflexly to adapt to their internal environment, resulting in the decrease of noradrenaline production at nerve endings. Our results support their view.

Hypertensives react more markedly to infused noradrenaline than normotensives. Not only noradrenaline but many other pressor substances are known to cause higher response in the former than in the latter. What role does this hyperresponsiveness play in the genesis of hypertension? And what is the mechanism that produces this hyperresponsiveness? Freedman ${ }^{12}$ and Tobian ${ }^{131}$ extensively investigated these problems, but they have not succeeded in arriving at a satisfactory answer.

It is desired these problems should be studied more profoundly in the future.

\section{SUMMARY}

The role of noradrenaline in essential hypertension was studied and the following results were obtained.

(1) Hypertensive subjects except the patient with pheochromocytoma 
were found more sensitive to the infusion of noradrenaline than normotensives. But in the cases with pheochromocytoma the sensitivity was weaker than in normotensives.

(2) There was no relation between the blood pressure and its response to Regitine.

(3) A negative correlation was observed between blood pressure and urinary excretion of noradrenaline.

From these experiments it may be concluded that hypertension (except in the case of pheochromocytoma) does not result from the excess of noradrenaline. Rather, in hypertensive subjects, it seems to be likely that the secretion of noradrenaline is reflexly decreased in order to adapt to their hypertensive state. On the other hand, the sensitivity to noradrenaline is obviously excessive in hypertensives. But the cause and the role of this hypersensitivity are entirely unknown to date.

These problems await future study.

\section{ACKNOWLEDGEMENT}

We are indebted to Prof. Dr. T. Torikai for his continuous leadership throughout these studies.

\section{References}

1) Goldenberg, M. et al, Am. J. Međ., 1919, 5, 792.

2) Goodman, L. S. \& Gilman, A., The Pharmacological Basis of Therapeuties, 2nd Ed. Macmillan Co., New York. p. 583, 1956.

3) Weil-Malherbe, H. \& Bone, A. D., Lancet, 1953, 1, 974.

4) Yoshinaga, K., Tohoku J. Exp. Med., 1959, 70, 261.

5) Euler, U.S.v., \& Floding, I., Acta Physiol. Scand., 1955, 33, (Suppl. 118) 57.

6) Euler, U.S.v. et al., Brit. J. Pharmacol., 1951, 6, 286.

7) Euler, U.S.v. et al., Scand. J. Clin. \& Lab. Invest., 1954, 6, 54.

8) Burn, G. P., Brit. Med. J., 1953, 1, 697.

9) Goldenberg, M. et al., J. Clin. Invest., 1951, 30, 641.

10) Sundin, T., Acta Med. Scand., 1956, 154, (Suppl. 313) 1.

11) M $\phi$ ler, P. et al., Scand. J. Clin. \& Lab. Invest., 1957, 9, 331.

12) Freedman, S.M. et al., Circulation Res., 1957, 5, 261.

13) Tohian, L. ef al., J. Lab. Clin. Med., 1959, 53, 842. 